University of Nebraska - Lincoln

DigitalCommons@University of Nebraska - Lincoln

Martin Centurion Publications

Research Papers in Physics and Astronomy

$1-2002$

\title{
Holographic recording of fast events on a CCD camera
}

Zhiwen Liu

California Institute of Technology

Martin Centurion

University of Nebraska - Lincoln, martin.centurion@unl.edu

George Panotopoulos

California Institute of Technology

John Hong

Rockwell Science Center, Thousand Oaks, California

Demetri Psaltis

California Institute of Technology

Follow this and additional works at: https://digitalcommons.unl.edu/physicscenturion

Part of the Physics Commons

Liu, Zhiwen; Centurion, Martin; Panotopoulos, George; Hong, John; and Psaltis, Demetri, "Holographic recording of fast events on a CCD camera" (2002). Martin Centurion Publications. 1.

https://digitalcommons.unl.edu/physicscenturion/1

This Article is brought to you for free and open access by the Research Papers in Physics and Astronomy at DigitalCommons@University of Nebraska - Lincoln. It has been accepted for inclusion in Martin Centurion Publications by an authorized administrator of DigitalCommons@University of Nebraska - Lincoln. 
This paper was published in Optics Letters 27, 22-24 (2002) and is made available as an electronic reprint with the permission of OSA. The paper can be found at the following URL on the OSA website: http://www.opticsinfobase.org/ol/abstract.cfm?URI=ol-27-1-22. Systematic or multiple reproduction or distribution to multiple locations via electronic or other means is prohibited and is subject to penalties under law.

\title{
Holographic recording of fast events on a CCD camera
}

\author{
Zhiwen Liu \\ Department of Electrical Engineering, California Institute of Technology, Pasadena, California 91125 \\ Martin Centurion \\ Department of Physics, California Institute of Technology, Pasadena, California 91125 \\ George Panotopoulos \\ Department of Electrical Engineering, California Institute of Technology, Pasadena, California 91125
}

John Hong

Rockwell Science Center, 1049 Camino Dos Rios, Thousand Oaks, California 91360

\section{Demetri Psaltis}

Department of Electrical Engineering, California Institute of Technology, Pasadena, California 91125

Received July 30, 2001

We report on holographic recording of nanosecond events on a conventional CCD camera. Three frames of an air-discharge event, with resolution of $5.9 \mathrm{~ns}$ and frame interval of $12 \mathrm{~ns}$, are recorded in a single CCD frame. Each individual frame is reconstructed by digital filtering of the CCD frame, since successively recorded holograms are centered at different carrier frequencies in the spatial frequency domain. (C) 2002 Optical Society of America

OCIS codes: $090.4220,140.3440,140.3540$.

Pulsed holograms can record an object field at selected times and thus serve as a useful tool for studying the dynamics of fast phenomena. A widely used technique is double-exposure interferometry, ${ }^{1}$ in which two pulsed holograms are recorded with identical reference pulses. During readout, the two signal beams are reconstructed simultaneously and interfere with each other. A sequence of holograms, i.e., a holographic movie, can be recorded by use of multiplexing techniques. In previous research, recording techniques included spatial ${ }^{2,3}$ and angular multiplexing. ${ }^{4,5}$ In recording a fast holographic movie, the sensitivity and response speed of the recording material are two very important issues. Because of the advancement of CCD technology, there has been a lot of interest in digital holography, ${ }^{6-8}$ in which holograms are recorded in a CCD camera and reconstructed digitally. CCD cameras not only provide a convenient interface with computers but also are an ideal thin recording material that is sensitive to a very broad spectrum ranging from infrared to ultraviolet and has high responsitivity (even hundreds of photons can be detected in one pixel). Multiple pulsed holograms were previously recorded on a single CCD frame ${ }^{9}$ by use of beam splitters to record three double-exposure holograms simultaneously. The separation between the two pulses that formed the double exposure was $150 \mu \mathrm{s}$. We use a special cavity to generate a pulse train and record a temporal sequence of holograms with a frame interval of $12 \mathrm{~ns}$.

As shown in Fig. 1, successive holograms are recorded with a slightly different reference angle as in angular multiplexing. Because the resolution of the CCD camera is typically $\sim 10 \mu \mathrm{m}$, a beam splitter is used to record holograms in a Mach-Zehnder interferometer configuration. The CCD camera records the integration of the whole sequence of pulse exposure. All the holograms are superimposed in one composite CCD frame, and each can be independently reconstructed through digital spatial filtering if the image bandwidth of each hologram is sufficiently low. One can then filter out individual holograms and reconstruct them by first performing a digital Fourier transform on the composite image, filtering a selected passband corresponding to the desired hologram, and then performing an inverse Fourier transform on the filtered result. As an example, we consider the interference pattern shown in Fig. 2a, which is a hologram of an air-discharge event. ${ }^{5}$ Figure $2 b$ shows the dc filtered Fourier transform. In general, the recorded pattern on the CCD is proportional to

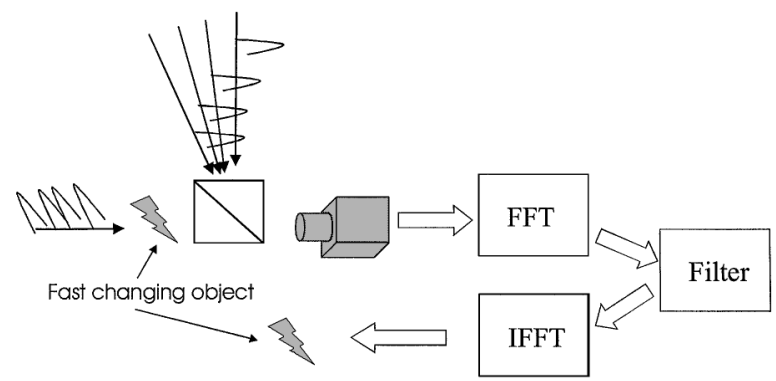

Fig. 1. Carrier multiplexing. FFT, fast Fourier transform; IFFT, inverse fast Fourier transform. 


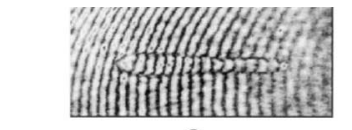

a

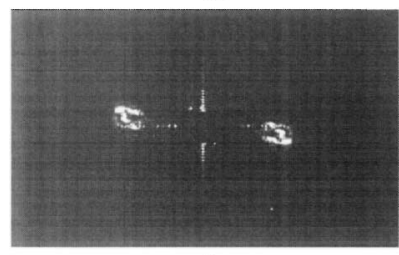

b

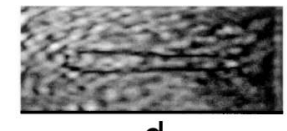

d

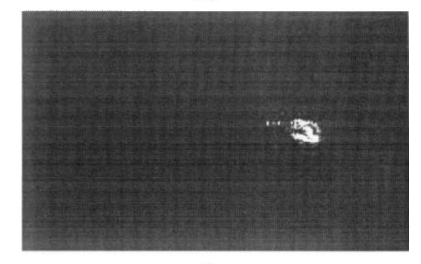

C
Fig. 2. Digital reconstruction of a CCD hologram: a, interference pattern; b, c, sidebands; d, reconstructed signal, $|S|$.

$$
I=|R+S|^{2}=|R|^{2}+|S|^{2}+R^{*} S+R S^{*},
$$

where $R$ is the plane-wave reference and $S$ is the signal. There are two sidebands in Fig. 2b, which correspond to the last two terms of Eq. (1). The position of the sideband is determined by the interference-pattern frequency or the carrier frequency, which one can change by changing the reference-beam incidence angle. We filter out one sideband, as shown in Fig. 2c, and perform the inverse Fourier transform. Either $R^{*} S$ or $R S^{*}$ is obtained. By taking the amplitude, we reconstruct the signal, $|S|$, in Fig. 2d. Different holograms can be recorded without overlapping in the frequency domain if the reference-beam angle or the carrier frequency of each hologram is different. The typical bandwidth of the air-discharge pattern is $\sim 0.0024 \mu \mathrm{m}^{-1}$. If the CCD pixel size is $10 \mu \mathrm{m}$ by $10 \mu \mathrm{m}$ (maximal bandwidth, $0.05 \mu \mathrm{m}^{-1}$ ), hundreds of frames can be recorded and reconstructed separately by means of two-dimensional carrier multiplexing.

Our experimental setup is shown in Fig. 3. A single pulse from the frequency-doubled $Q$-switched Nd:YAG laser (wavelength, $532 \mathrm{~nm}$; pulse width, $5.9 \mathrm{~ns}$; energy per pulse, $300 \mathrm{~mJ}$; beam diameter, $9 \mathrm{~mm}$ ) is coupled into the multiple-pulse-generation cavity ${ }^{5}$ by a small coupling mirror. After the coupling mirror, the pulse travels as if it had originated from the center of the front mirror. Since the two lenses in the cavity form an imaging system, the pulse would be blocked by the coupling mirror from retracing the previous path if the two cavity mirrors were parallel to each other. We break the symmetry of the cavity by slanting the rear mirror slightly, such that the pulse just misses the coupling mirror. Multiple pulses propagating in different directions can be generated if the front mirror is partially reflecting. The pulse interval depends on the round-trip time of the cavity, and we can change the interval by tuning the cavity length. The generated pulses are relayed by another imaging system and split into a reference and a signal by a nonpolarizing beam splitter. We recombine the reference and the signal by use of mirrors and then record holograms on a
CCD camera (Pulnix TM-7EX; 30 frames/s; $768 \times 494$ pixels; pixel size, $8.4 \mu \mathrm{m} \times 9.8 \mu \mathrm{m}$ ). As shown in Fig. 3, a portion of the laser pulse is split off and focused on the signal path to induce the air discharge. Holograms of the optical breakdown in air $^{10}$ are then recorded with this setup.

We generated three pulses with pulse separation of $12 \mathrm{~ns}$. The three plane-wave pulsed holograms are given in Fig. 4a, and the dc filtered Fourier-transform spectrum is shown in Fig. 4b. Clearly, the holograms are well separated in the frequency domain. We then recorded an air-discharge movie. The holograms and the Fourier transform of the composite CCD frame are shown in Figs. 4c and 4d, respectively. We reconstructed the three holograms, and the results are given in Figs. 5a-5c. Because the holograms are recorded in the far field, we need to compensate for the diffraction of the image. Consider one of the holograms, $R_{i}{ }^{*} S_{i}$. We take the $x-y$ plane as the plane of the CCD camera. We can calculate the field at $z$ by convolving the field at $z=0$ with the Fresnel diffraction kernel, ${ }^{11}$ and the field is then given by

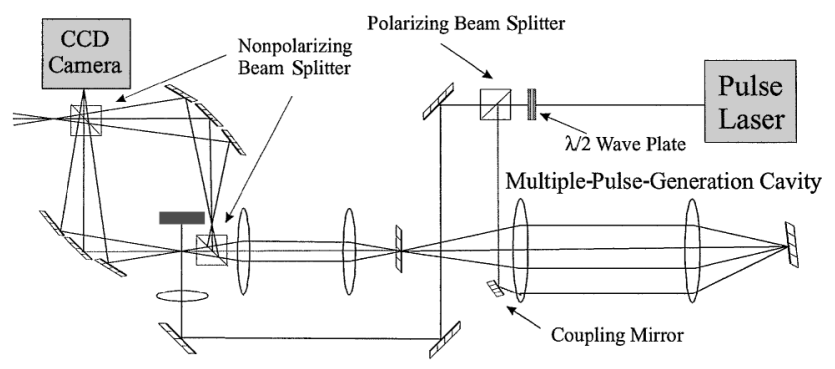

Fig. 3. Experimental setup.

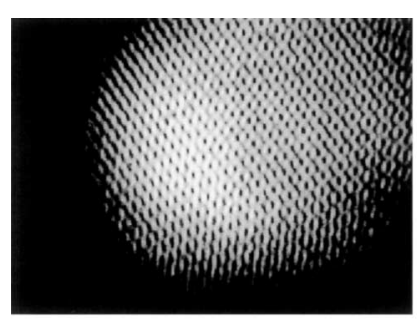

a

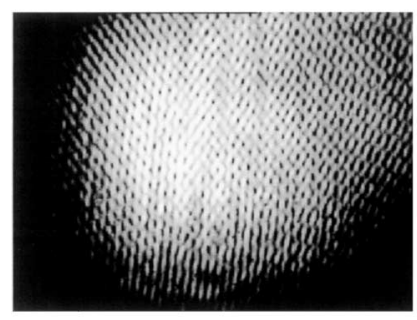

C

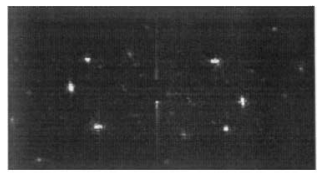

b

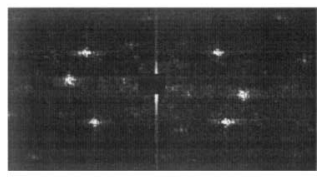

d
Fig. 4. Pulsed holograms recorded on a CCD camera. $\mathrm{a}$ and $\mathrm{b}$ are the plane-wave holograms and the corresponding dc filtered Fourier transform, respectively. $\mathrm{c}$ and $\mathrm{d}$ are the holograms and Fourier transform, respectively, of an air-discharge event. Three frames are recorded, and the frame interval is $12 \mathrm{~ns}$. 


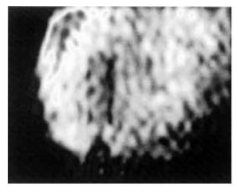

a

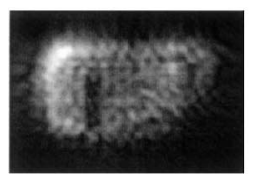

d

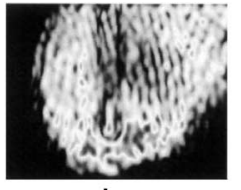

b

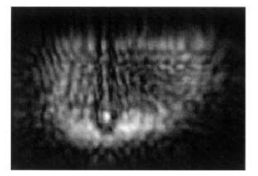

e

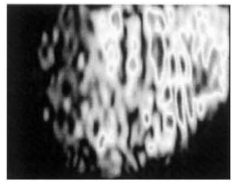

C

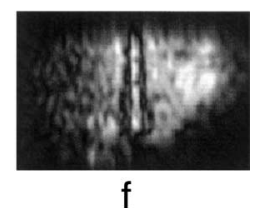

Fig. 5. Frame reconstruction: a-c, reconstructed frames without diffraction compensation; $d-f$, frames that were digitally compensated for diffraction.

$$
\begin{aligned}
& S_{i}{ }^{\prime}(x, y, z)= \iint R_{i}{ }^{*}\left(x_{1}, y_{1}\right) S_{i}\left(x_{1}, y_{1}\right) \\
& \times \exp \left\{j \frac{\pi}{\lambda z}\left[\left(x-x_{1}\right)^{2}+\left(y-y_{1}\right)^{2}\right]\right\} \mathrm{d} x_{1} \mathrm{~d} y_{1} \\
&= \exp \left[j \frac{\pi}{\lambda z}\left(x^{2}+y^{2}\right)\right] \iint R_{i}^{*}\left(x_{1}, y_{1}\right) S_{i}\left(x_{1}, y_{1}\right) \\
& \times \exp \left[j \frac{\pi}{\lambda z}\left(x_{1}{ }^{2}+y_{1}{ }^{2}\right)\right] \exp \left[-j \frac{2 \pi}{\lambda z}\left(x x_{1}+y y_{1}\right)\right] \mathrm{d} x_{1} \mathrm{~d} y_{1} .
\end{aligned}
$$

We need only to multiply the reconstructed field by a chirped phase term and then perform the Fourier transform. The presence of the reference $R_{i}{ }^{*}$ merely shifts the position of the image slightly from the center. The diffraction can be compensated for by use of a negative value of $z$ for $R_{i}{ }^{*} S_{i}$ or a positive value of $z$ for the $R_{i} S_{i}{ }^{*}$ term. The three frames after the diffraction compensation are shown in Figs. 5d-5f. In the frame 5 (d) the plasma created by the optical breakdown in the air is shown, and in (5e) and (5f) a shock wave is formed and propagates outward.

In summary, we have demonstrated a system for recording fast movies on a conventional CCD camera with speed comparable to the state-of-the-art multiintensified CCD system (8 frames; resolution, $10 \mathrm{~ns}$; frame interval, $10 \mathrm{~ns}$; see, e.g., Ref. 12). More importantly, our method can also be extended to the femtosecond regime if a femtosecond pulsed laser is used. A typical Ti:sapphire mode-locked laser has pulse energy of a few nanojoules. Let us consider recording a movie by use of a single femtosecond pulse with 1-nJ energy and beam size of $1 \mathrm{~cm}^{2}$. We estimate that, if the pulse uniformly illuminates the CCD, then each pixel has $\sim 4000$ photons. Since we need only several hundred photons per pixel for recording of a single hologram, we expect that as many as 10 frames can be recorded with a single 1-nJ pulse. An optical amplifier is required for longer movies. As the number of frames is increased, the maximum space-bandwidth product of the images that are reconstructed is proportionally reduced.

This work was supported by the U.S. Air Force Office of Scientific Research and the National Science Foundation Neuromorphic Engineering Research Center. We thank Gregory J. Steckman for his contributions in the previous stages of this work, from which we benefited greatly. Z. Liu's e-mail address is zliu@sunoptics.caltech.edu.

\section{References}

1. L. O. Heflinger, R. F. Wuerker, and R. E. Brooks, "Holographic interferometry," J. Appl. Phys. 37, 642649 (1966).

2. T. Tschudi, C. Yamanaka, T. Sasaki, K. Yoshida, and K. Tanaka, "A study of high-power laser effects in dielectrics using multiframe picosecond holography," J. Phys. D 11, 177-180 (1978).

3. M. J. Ehrlich, J. S. Steckenrider, and J. W. Wagner, "System for high-speed time-resolved holography of transient events," Appl. Opt. 31, 5947-5951 (1992).

4. S. Suzuki, Y. Nozaki, and H. Kimura, "High-speed holographic microscopy for fast-propagating cracks in transparent materials," Appl. Opt. 36, 7224-7233 (1997).

5. Z. Liu, G. Steckman, and D. Psaltis, "Holographic recordings of fast phenomena," submitted to Appl. Phys. Lett.

6. E. Cuche, F. Bevilacqua, and C. Depeursinge, "Digital holography for quantitative phase-contrast imaging," Opt. Lett. 24, 291-293 (1999).

7. G. Indebetouw and P. Klysubun, "Space-time digital holography: A three-dimensional microscopic imaging scheme with an arbitrary degree of spatial coherence," Appl. Phys. Lett. 75, 2017-2019 (1999).

8. S. Schedin, G. Pedrini, H. J. Tiziani, A. K. Aggarwal, and M. E. Gusev, "Highly sensitive pulsed digital holography for built-in defect analysis with a laser excitation," Appl. Opt. 40, 100-103 (2001).

9. S. Schedin, G. Pedrini, H. J. Tiziani, and F. M. Santoyo, "Simultaneous three-dimensional dynamic deformation measurements with pulsed digital holography," Appl. Opt. 38, 7056-7062 (1999).

10. H. Sobral, M. Villagran-Muniz, R. Navarro-Gonzalez, and A. C. Raga, "Temporal evolution of the shock wave and hot core air in laser induced plasma," Appl. Phys. Lett. 77, 3158-3160 (2000).

11. J. Goodman, Introduction to Fourier Optics (McGraw-Hill, New York, 1996).

12. http://www.drs.com. 